Article

\title{
Synthesis of 2-Oxazolines from Ring Opening Isomerization of 3-Amido-2-Phenyl Azetidines
}

\author{
Xin Zhou, Baiyi Mao and Zhanbin Zhang *(D) \\ College of Chemistry, Beijing Normal University, Beijing 100875, China; 201321150129@mail.bnu.edu.cn (X.Z.); \\ 201821150037@mail.bnu.edu.cn (B.M.) \\ * Correspondence: zhangzb@bnu.edu.cn; Tel.: +86-10-5880-6051
}

check for

updates

Citation: Zhou, X.; Mao, B.; Zhang, Z. Synthesis of 2-Oxazolines from Ring Opening Isomerization of 3-Amido-2-Phenyl Azetidines. Molecules 2021, 26, 857. https://doi. org/10.3390/molecules26040857

Academic Editor: Paola Vitale

Received: 17 January 2021

Accepted: 3 February 2021

Published: 6 February 2021

Publisher's Note: MDPI stays neutral with regard to jurisdictional claims in published maps and institutional affiliations.

Copyright: (c) 2021 by the authors. Licensee MDPI, Basel, Switzerland. This article is an open access article distributed under the terms and conditions of the Creative Commons Attribution (CC BY) license (https:// creativecommons.org/licenses/by/ $4.0 /)$.
Abstract: Chiral 2-oxazolines are valuable building blocks and famous ligands for asymmetric catalysis. The most common synthesis involves the reaction of an amino alcohol with a carboxylic acid. In this paper, an efficient synthesis of 2-oxazolines has been achieved via the stereospecific isomerization of 3-amido-2-phenyl azetidines. The reactions were studied in the presence of both Brønsted and Lewis acids, and $\mathrm{Cu}(\mathrm{OTf})_{2}$ was found to be the most effective.

Keywords: azetidine; oxazoline; isomerization; amide; acid

\section{Introduction}

2-Oxazolines are very important five-membered heterocycles existing in numerous medicinally active compounds and natural products [1-6]. They are also widely applied as synthetic intermediates, protecting, activating and directing groups in organic synthesis. In addition, the optically active 2-oxazolines are valuable chiral building blocks and famous ligands for asymmetric catalysis [7-11]. There are numerous methods that have been developed for the preparation of 2-oxazolines [12-15]. The most common process for their preparation involves the reaction of an amino alcohol with a carboxylic acid [16,17]. In this way, oxazoline amines can be accessed from commercially available $\beta$-amino alcohols and suitably protected $\alpha$-amino acids [18].

Recently, we reported the synthesis of chiral 4,5-dihydrothiazol-2-amines and 4,5dihydrooxazol-2-amines through an unexpected ring opening reaction of azetidines [19]. Based on this strategy, we now extend our research work and report herein the asymmetric synthesis of 2-oxazolines via the stereospecific isomerization of 3-amido-2-phenyl azetidines in the presence of acid (Scheme 1).

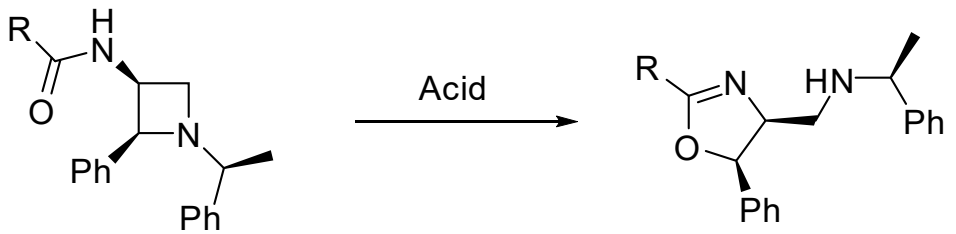

Scheme 1. Isomerization of 3-amido-2-phenyl azetidines to 2-oxazolines.

\section{Results}

Chiral 3-amino-2-phenyl azetidine 1 was prepared as previously reported by our group [20]. The coupling of 3-amino-2-phenyl azetidine 1 with acids 2 led to the corresponding amides 3, which were obtained in 77\% to 95\% yields (Scheme 2; see Supplementary Materials).

The isomerization of amide 3a was examined in different conditions (Scheme 3, Table 1). The results (Table 1) showed that the isomerization of 3a did not occur at reflux in 1,2dichloroethane (Table 1, entry 1 ) or in toluene (Table 1, entry 2) without additive or in the 
presence of bases such as DABCO, t-BuOK and $\mathrm{NaH}$ (Table 1, entries 3-5). Nevertheless, isomerization of amide 3a to 2-oxazoline $4 \mathbf{a}$ occurred in the presence of acids, including Lewis acids and Brønsted acids but excepting acetic acid (Table 1, entries 6-16). In search for a better additive, we explored Brønsted acids such as $\mathrm{HClO}_{4}, \mathrm{CF}_{3} \mathrm{SO}_{3} \mathrm{H}, \mathrm{CH}_{3} \mathrm{COOH}$ and $\mathrm{CF}_{3} \mathrm{COOH}$ (TFA). It was found that TFA was the suitable additive for this transformation and the yield of 4 a could achieve up to $91 \%$ in 1,2-dichloroethane for about half an hour (Table 1, entry 10). However, prolonging the reaction time to over $2 \mathrm{~h}$ resulted in some degradation (Table 1, entry 11). Moreover, among the Lewis acids, $\mathrm{Cu}(\mathrm{OTf})_{2}$ turned out to be the most efficient for this transformation, allowing the formation of 4 a in a $93 \%$ yield (Table 1, entry 14).

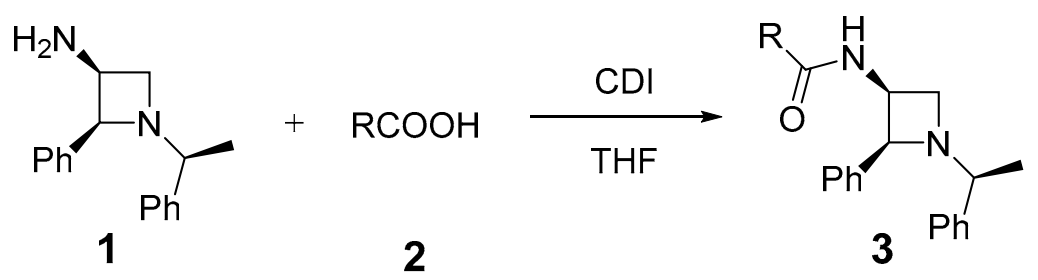

Scheme 2. Synthesis of 3-amido-2-phenyl azetidines

Table 1. Isomerization of amide $3 \mathbf{a}^{1}$.

\begin{tabular}{|c|c|c|c|c|}
\hline Entry & $\begin{array}{l}\text { Additive } \\
\text { (Equiv.) }\end{array}$ & Solvent & Time (h) & Yield $(\%)^{2}$ \\
\hline 1 & - & $\mathrm{ClCH}_{2} \mathrm{CH}_{2} \mathrm{Cl}$ & 6 & n.r. \\
\hline 2 & - & Toluene & 6 & n.r. \\
\hline 3 & $\mathrm{DABCO}(1)$ & $\mathrm{ClCH}_{2} \mathrm{CH}_{2} \mathrm{Cl}$ & 6 & n.r. \\
\hline 4 & $\mathrm{t}-\mathrm{BuOK}(1)$ & THF & 6 & n.r. \\
\hline 5 & $\mathrm{NaH}(1)$ & THF & 6 & n.r. \\
\hline 6 & $\mathrm{HClO}_{4}(1)$ & $\mathrm{ClCH}_{2} \mathrm{CH}_{2} \mathrm{Cl}$ & 0.5 & 87 \\
\hline 7 & $\mathrm{CF}_{3} \mathrm{SO}_{3} \mathrm{H}(1)$ & $\mathrm{ClCH}_{2} \mathrm{CH}_{2} \mathrm{Cl}$ & 0.5 & 65 \\
\hline 8 & $\mathrm{CH}_{3} \mathrm{COOH}(1)$ & $\mathrm{CH}_{2} \mathrm{Cl}_{2}$ & 12 & n.r. \\
\hline 9 & $\mathrm{CF}_{3} \mathrm{COOH}(1)$ & $\mathrm{CH}_{2} \mathrm{Cl}_{2}$ & 12 & 37 \\
\hline 10 & $\mathrm{CF}_{3} \mathrm{COOH}(1)$ & $\mathrm{ClCH}_{2} \mathrm{CH}_{2} \mathrm{Cl}$ & 0.5 & 91 \\
\hline 11 & $\mathrm{CF}_{3} \mathrm{COOH}(1)$ & $\mathrm{ClCH}_{2} \mathrm{CH}_{2} \mathrm{Cl}$ & 2 & 86 \\
\hline 12 & $\mathrm{CF}_{3} \mathrm{COOH}(0.75)$ & $\mathrm{ClCH}_{2} \mathrm{CH}_{2} \mathrm{Cl}$ & 0.5 & 71 \\
\hline 13 & TMSOTf (1) & $\mathrm{ClCH}_{2} \mathrm{CH}_{2} \mathrm{Cl}$ & 4 & 72 \\
\hline 14 & $\mathrm{Cu}(\mathrm{OTf})_{2}(1)$ & $\mathrm{ClCH}_{2} \mathrm{CH}_{2} \mathrm{Cl}$ & 4 & 93 \\
\hline 15 & $\mathrm{Cu}(\mathrm{OTf})_{2}(0.5)$ & $\mathrm{ClCH}_{2} \mathrm{CH}_{2} \mathrm{Cl}$ & 4 & 90 \\
\hline 16 & $\mathrm{BF}_{3} \cdot \mathrm{Et}_{2} \mathrm{O}(1)$ & $\mathrm{ClCH}_{2} \mathrm{CH}_{2} \mathrm{Cl}$ & 6 & 87 \\
\hline
\end{tabular}

${ }^{1}$ The reactions were conducted with amide $3 \mathbf{a}(1 \mathrm{mmol})$ in solvent $(10 \mathrm{~mL})$ in the presence of additive at reflux.

${ }^{2}$ Isolated yields.<smiles>C[C@H](c1ccccc1)N1C[C@H](NC(=O)c2ccccc2)[C@H]1c1ccccc1</smiles>

$3 a$<smiles>C[C@H](NC[C@H]1N=C(c2ccccc2)O[C@H]1c1ccccc1)c1ccccc1</smiles>

$4 a$

Scheme 3. Isomerization of amide 3a in different conditions.

After optimizing the reaction conditions, we extended the substrate scope and different amides were examined (Scheme 4, Table 2). The amides with aryl, heteroaryl and alkyl groups were successfully isomerized to 2-oxazolines in the presence of $\mathrm{Cu}(\mathrm{OTf})_{2}$ in high yields (Table 2, entries 1-9 and 13-16). However, those substrates with 2-hydroxyl or 2amino groups could not be isomerized, presumably due to their coordination to $\mathrm{Cu}(\mathrm{OTf})_{2}$. 
Nevertheless, these substrates could be isomerized to 2-oxazolines in the presence of $\mathrm{CF}_{3} \mathrm{COOH}$ in good yields (Table 2, entries 10-12).<smiles>[R]C(=O)N[C@@H]1CN(C(C)c2ccccc2)C1c1ccccc1</smiles>

3<smiles>[O-]C(Cl)(Cl)OCCCl</smiles><smiles>[R]C1=NC(CNC(C)c2ccccc2)C(P)O1</smiles>

4

Scheme 4. Substrate scope of the isomerization of amides 3.

Table 2. Isomerizations of various amides in the presence of $\mathrm{Cu}(\mathrm{OTf})_{2}{ }^{1}$.

\begin{tabular}{|c|c|c|c|}
\hline Entry & $\mathbf{R}$ & Product & Yield (\%) ${ }^{2}$ \\
\hline 1 & 3a: $\mathrm{C}_{6} \mathrm{H}_{5}^{-}$ & $4 a$ & 96 \\
\hline 2 & 3b: $4-\mathrm{MeOC}_{6} \mathrm{H}_{4}^{-}$ & $4 b$ & 94 \\
\hline 3 & 3c: $2-\mathrm{MeOC}_{6} \mathrm{H}_{4}^{-}$ & $4 c$ & 93 \\
\hline 4 & 3d: $4-\mathrm{O}_{2} \mathrm{NC}_{6} \mathrm{H}_{4}^{-}$ & $4 d$ & 94 \\
\hline 5 & 3e: $2-\mathrm{O}_{2} \mathrm{NC}_{6} \mathrm{H}_{4}^{-}$ & $4 e$ & 91 \\
\hline 6 & 3f: $4-\mathrm{ClC}_{6} \mathrm{H}_{4}^{-}$ & $4 f$ & 79 \\
\hline 7 & $3 \mathrm{~g}: 2-\mathrm{ClC}_{6} \mathrm{H}_{4}^{-}$ & $4 g$ & 85 \\
\hline 8 & 3h: 4-pyridyl & $4 h$ & 82 \\
\hline 9 & 3i: 2-pyridyl & $4 i$ & 87 \\
\hline $10^{3}$ & $3 \mathrm{j}: 2-\mathrm{HOC}_{6} \mathrm{H}_{4}-$ & $4 \mathbf{j}$ & 87 \\
\hline $11^{3}$ & $3 \mathbf{k}: 2-\mathrm{H}_{2} \mathrm{NC}_{6} \mathrm{H}_{4}-$ & $4 k$ & 82 \\
\hline $12^{3}$ & 31: 1-hydroxynaphthalen-2-yl & 41 & 88 \\
\hline 13 & 3m: thiophen-2-yl & $4 \mathrm{~m}$ & 90 \\
\hline 14 & 3n: furan-2-yl & $4 n$ & 84 \\
\hline 15 & 3o: $\mathrm{Me}-$ & 40 & 85 \\
\hline 16 & 3p: $\mathrm{C}_{6} \mathrm{H}_{5} \mathrm{CH}_{2-}^{-}$ & $4 p$ & 83 \\
\hline
\end{tabular}

${ }^{1}$ Reactions were conducted with amide $3(1 \mathrm{mmol})$ at reflux for $4 \mathrm{~h}$ in $\mathrm{ClCH}_{2} \mathrm{CH}_{2} \mathrm{Cl}(10 \mathrm{~mL})$ in the presence of $\mathrm{Cu}(\mathrm{OTf})_{2}(0.5 \mathrm{mmol}){ }^{2}$ Isolated yields. ${ }^{3}$ Reactions were conducted in the presence of $\mathrm{CF}_{3} \mathrm{COOH}(1.5 \mathrm{mmol})$ at reflux for $30 \mathrm{~min}$.

A proposed mechanism for this transformation is shown below (Scheme 5). The isomerization of amides 3 occurred regiospecifically by presumably an $\mathrm{SN}_{2}$ nucleophilic attack at the more active $\mathrm{C} 2$ but not the less-hindered $\mathrm{C} 4$ of the azetidine ring, thus the stereochemistry of 2-oxazolines 4 was shown to be cis [21]. This is also supported by comparison of the coupling constant $(10 \mathrm{~Hz})$ between $\mathrm{H} 4$ and $\mathrm{H} 5$ with reported cis-2oxazolines [22,23]. In addition, all the structures of 2-oxazolines were established by ${ }^{1} \mathrm{H}$ and ${ }^{13} \mathrm{C}$ NMR, high resolution mass spectra (HRMS), IR. Furthermore, the absolute configuration of 41 was further confirmed by $\mathrm{X}$-ray analysis (Figure 1).

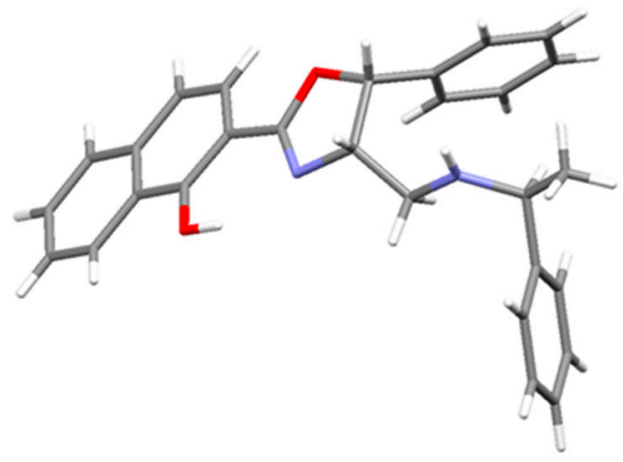

Figure 1. Structure of 41 . 
<smiles>[R]C(=O)N[C@@H]1C[NH+](C(C)c2ccccc2)[C@H]1c1ccccc1</smiles>

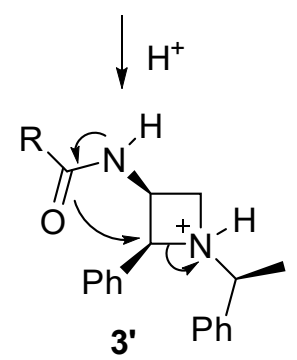<smiles>[R]C1=N[C@H](CNC(C)c2ccccc2)[C@H](P)O1</smiles><smiles></smiles><smiles>[R]C1=[NH+][C@@H](CNC(C)c2ccccc2)[C@H](P)O1</smiles>

Scheme 5. Proposed mechanism.

\section{Materials and Methods}

\subsection{General Information}

All reactants and reagents were commercially available and were used without further purification. ${ }^{1} \mathrm{H}$ and ${ }^{13} \mathrm{C}$ NMR spectra were recorded on a Bruker Advance III $400 \mathrm{MHz}$ spectrometer (Billerica, MA, USA). Chemical shifts are reported in $\delta$ values (ppm) relative to an internal reference $(0.03 \% \mathrm{v} / \mathrm{v})$ of tetramethylsilane (TMS) for ${ }^{1} \mathrm{H}$ NMR or the solvent signal, chloroform $\left(\mathrm{CDCl}_{3}\right)$, for ${ }^{13} \mathrm{C}$ NMR. IR data was obtained with an IRAffinity-1 spectrometer (Shimadzu, Kyoto, Japan). High resolution mass spectrometry (HRMS) was conducted with a high-resolution LCT Premier XE mass spectrometer in positive ESI mode (Waters, MA, USA). Melting points were measured on a digital melting point apparatus and are uncorrected.

\subsection{General Procedure for the Isomeization of Amide 3}

A mixture of amide $3(1 \mathrm{mmol}), \mathrm{Cu}(\mathrm{OTf})_{2}(0.5 \mathrm{mmol})$ in 1,2-dichloroethane $(10 \mathrm{~mL})$ was refluxed for $4 \mathrm{~h}$. The mixture was washed with water $(10 \mathrm{~mL})$, saturated $\mathrm{NaHCO}_{3}$ $(10 \mathrm{~mL})$, and dried over $\mathrm{Na}_{2} \mathrm{SO}_{4}$. The solvent was removed under reduced pressure. The residue was purified by gradient column chromatography on silica gel with PE/EA (5:1-2:1) as eluent to give oxazoline 4 .

(S)-N-(((4S,5R)-2,5-diphenyl-4,5-dihydrooxazol-4-yl)methyl)-1-phenylethanamine (4a)

Yellow oil, 96\% yield, $[\alpha]_{\mathrm{D}}{ }^{20}=-322.0\left(\mathrm{c} 1.0 \mathrm{CH}_{3} \mathrm{COOC}_{2} \mathrm{H}_{5}\right) ;{ }^{1} \mathrm{H} \mathrm{NMR}(400 \mathrm{MHz}$, $\left.\mathrm{CDCl}_{3}\right) \delta=8.02(\mathrm{~d}, J=7.4 \mathrm{~Hz}, 2 \mathrm{H}), 7.59-7.00(\mathrm{~m}, 13 \mathrm{H}), 5.79(\mathrm{~d}, J=9.9 \mathrm{~Hz}, 1 \mathrm{H}), 4.68(\mathrm{q}$, $J=7.2 \mathrm{~Hz}, 1 \mathrm{H}), 3.46(\mathrm{q}, J=6.4 \mathrm{~Hz}, 1 \mathrm{H}), 2.43-2.14(\mathrm{~m}, 3 \mathrm{H}), 1.16(\mathrm{~d}, J=6.5 \mathrm{~Hz}, 3 \mathrm{H}) ;{ }^{13} \mathrm{C}$ $\operatorname{NMR}\left(100 \mathrm{MHz} \mathrm{CDCl}_{3}\right) \delta=23.72,49.35,58.29,69.67,83.13,126.36,126.49,126.89,127.53$, $128.21,128.35,128.38,128.42,128.44,131.60,136.41,144.86,163.76$; IR (KBr) v 3061, 3028, $2963,2924,1651,1603,1580,1495,1450,1368,1333,1277,1246,1209,1175,1128,1082,1065$, $1024,964,781,760,696,540 \mathrm{~cm}^{-1}$; HRMS $\mathrm{m} / z[\mathrm{M}+\mathrm{H}]^{+}$calcd. for $\left[\mathrm{C}_{24} \mathrm{H}_{25} \mathrm{~N}_{2} \mathrm{O}\right]^{+} 357.1961$, found 357.1962.

(S)-N-(((4S,5R)-2-(4-methoxyphenyl)-5-phenyl-4,5-dihydrooxazol-4-yl)methyl)-1-phenylethana mine $(4 b)$

Yellow solid, 94\% yield, mp:71 74 ${ }^{\circ} \mathrm{C},[\alpha]_{\mathrm{D}}^{20}=-273.8\left(\mathrm{c} 1.0 \mathrm{CH}_{3} \mathrm{COOC}_{2} \mathrm{H}_{5}\right) ;{ }^{1} \mathrm{H}$ NMR $\left(400 \mathrm{MHz}, \mathrm{CDCl}_{3}\right) \delta=7.96(\mathrm{~d}, J=8.8 \mathrm{~Hz}, 2 \mathrm{H}), 7.37-7.07(\mathrm{~m}, 10 \mathrm{H}), 6.92(\mathrm{~d}, J=8.9 \mathrm{~Hz}, 2 \mathrm{H})$, $5.73(\mathrm{~d}, J=9.9 \mathrm{~Hz}, 1 \mathrm{H}), 4.61(\mathrm{dt}, J=9.8,7.0 \mathrm{~Hz}, 1 \mathrm{H}), 3.81(\mathrm{~s}, 3 \mathrm{H}), 3.41(\mathrm{q}, J=6.5 \mathrm{~Hz}, 1 \mathrm{H})$, $2.28(\mathrm{~d}, J=7.0 \mathrm{~Hz}, 2 \mathrm{H}), 1.69(\mathrm{br} \mathrm{s}, 1 \mathrm{H}), 1.12(\mathrm{~d}, J=6.6 \mathrm{~Hz}, 3 \mathrm{H}) ;{ }^{13} \mathrm{C} \mathrm{NMR}\left(100 \mathrm{MHz}, \mathrm{CDCl}_{3}\right)$ $\delta=23.99,49.55,55.38,58.23,69.85,83.10,113.80,120.08,126.42,126.46,126.74,128.12$, $128.31,128.33,130.16,136.69,145.50,162.28,163.51$; IR (KBr) v 3061, 3026, 2961, 2930, 2837, 
$1647,1609,1512,1495,1452,1420,1368,1335,1310,1256,1167,1076,1030,966,841,741,700$, $685 \mathrm{~cm}^{-1} ;$ HRMS $m / z[\mathrm{M}+\mathrm{H}]^{+}$calcd. for $\left[\mathrm{C}_{25} \mathrm{H}_{27} \mathrm{~N}_{2} \mathrm{O}_{2}\right]^{+} 387.2067$, found 387.2067.

(S)-N-(((4S,5R)-2-(2-methoxyphenyl)-5-phenyl-4,5-dihydrooxazol-4-yl)methyl)-1-phenylethana mine $(4 \mathrm{c})$

Yellow oil, 93\% yield, $[\alpha]_{\mathrm{D}}{ }^{20}=-204.5\left(\mathrm{c} 1.0 \mathrm{CH}_{3} \mathrm{COOC}_{2} \mathrm{H}_{5}\right) ;{ }^{1} \mathrm{H} \mathrm{NMR}(400 \mathrm{MHz}$, $\left.\mathrm{CDCl}_{3}\right) \delta=7.80(\mathrm{~d}, J=7.2 \mathrm{~Hz}, 1 \mathrm{H}), 7.44(\mathrm{t}, J=7.3 \mathrm{~Hz}, 1 \mathrm{H}), 7.39-7.06(\mathrm{~m}, 10 \mathrm{H}), 6.98(\mathrm{~d}, J$ $=7.9 \mathrm{~Hz}, 2 \mathrm{H}), 5.76(\mathrm{~d}, J=9.9 \mathrm{~Hz}, 1 \mathrm{H}), 4.80-4.74(\mathrm{~m}, 1 \mathrm{H}), 3.90(\mathrm{~s}, 4 \mathrm{H}), 3.52-3.45(\mathrm{~m}, 1 \mathrm{H})$, 2.39-2.27 (m, 2H), $1.21(\mathrm{~d}, J=6.1 \mathrm{~Hz}, 3 \mathrm{H}) ;{ }^{13} \mathrm{C} \mathrm{NMR}\left(100 \mathrm{MHz}, \mathrm{CDCl}_{3}\right) \delta=23.31,49.09$, $55.99,58.38,69.43,82.44,111.71,116.89,120.35,126.45,126.64,127.10,128.13,128.32,128.41$, 131.40, 132.58, 136.43, 143.87, 158.61, 162.87; IR (KBr) v 3061, 3028, 2961, 2924, 2837, 1653, 1636, 1578, 1560, 1491, 1458, 1437, 1331, 1283, 1258, 1123, 1043, 1024, 968, 754, 700, $592 \mathrm{~cm}^{-1}$; HRMS $m / z[\mathrm{M}+\mathrm{H}]^{+}$calcd. for $\left[\mathrm{C}_{25} \mathrm{H}_{27} \mathrm{~N}_{2} \mathrm{O}_{2}\right]^{+}$387.2067, found 387.2064.

(S)-N-((4S,5R)-2-(4-nitrophenyl)-5-phenyl-4,5-dihydrooxazol-4-yl)methyl)-1-phenylethana mine $(4 \mathrm{~d})$

White solid, 94\% yield, mp: $68 \sim 71{ }^{\circ} \mathrm{C},[\alpha]_{\mathrm{D}}{ }^{20}=-145.8\left(\mathrm{c} 1.0 \mathrm{CH}_{3} \mathrm{COOC}_{2} \mathrm{H}_{5}\right) ;{ }^{1} \mathrm{H} \mathrm{NMR}$ $\left(400 \mathrm{MHz} \mathrm{CDCl}_{3}\right) \delta=8.29(\mathrm{~d}, J=8.9 \mathrm{~Hz}, 2 \mathrm{H}), 8.19(\mathrm{~d}, J=8.9 \mathrm{~Hz}, 2 \mathrm{H}), 7.42-7.30(\mathrm{~m}, 3 \mathrm{H})$, 7.28-7.11 (m, 7H), $5.85(\mathrm{~d}, J=10.1 \mathrm{~Hz}, 1 \mathrm{H}), 4.72(\mathrm{dt}, J=10.0,7.1 \mathrm{~Hz}, 1 \mathrm{H}), 3.45(\mathrm{q}, J=6.5 \mathrm{~Hz}$, 1H), 2.38-2.21 (m, 2H), 2.02 (br s, $1 \mathrm{H}), 1.16(\mathrm{~d}, J=6.6 \mathrm{~Hz}, 3 \mathrm{H}) ;{ }^{13} \mathrm{C} \mathrm{NMR}\left(100 \mathrm{MHz}, \mathrm{CDCl}_{3}\right)$ $\delta=23.72,49.16,58.25,70.20,83.83,123.62,126.28,126.41,126.91,128.38,128.44,128.46$, $129.42,133.31,135.79,144.99,149.64,161.93$; IR (KBr) v 3082, 3061, 3030, 2959, 2924, 2873, 1653, 1647, 1599, 1524, 1493, 1452, 1410, 1348, 1277, 1107, 1076, 1014, 961, 866, 851, 762, $702 \mathrm{~cm}^{-1} ;$ HRMS $m / z[\mathrm{M}+\mathrm{H}]^{+}$calcd. for $\left[\mathrm{C}_{24} \mathrm{H}_{24} \mathrm{~N}_{3} \mathrm{O}_{3}\right]^{+} 402.1812$, found 402.1812 .

(S)-N-(((4S,5R)-2-(2-nitrophenyl)-5-phenyl-4,5-dihydrooxazol-4-yl)methyl)-1-phenylethana mine (4e)

White solid, 91\% yield, mp: $44 \sim 47^{\circ} \mathrm{C},[\alpha]_{\mathrm{D}}{ }^{20}=-314.7\left(\mathrm{c} 1.0 \mathrm{CH}_{3} \mathrm{COOC}_{2} \mathrm{H}_{5}\right) ;{ }^{1} \mathrm{H} \mathrm{NMR}$ $\left(400 \mathrm{MHz} \mathrm{CDCl}_{3}\right) \delta=7.90-7.79(\mathrm{~m}, 2 \mathrm{H}), 7.68-7.53(\mathrm{~m}, 2 \mathrm{H}), 7.39-7.06(\mathrm{~m}, 10 \mathrm{H}), 5.80(\mathrm{~d}$, $J=10.0 \mathrm{~Hz}, 1 \mathrm{H}), 4.66(\mathrm{dt}, J=9.8,7.0 \mathrm{~Hz}, 1 \mathrm{H}), 3.44(\mathrm{q}, J=6.5 \mathrm{~Hz}, 1 \mathrm{H}), 2.44-2.12(\mathrm{~m}, 2 \mathrm{H}), 1.47$ (br s, 1H), $1.12(\mathrm{~d}, J=6.6 \mathrm{~Hz}, 3 \mathrm{H}) ;{ }^{13} \mathrm{C} \mathrm{NMR}\left(100 \mathrm{MHz}, \mathrm{CDCl}_{3}\right) \delta 24.00,49.00,58.11,69.98$, $84.57,123.14,123.96,126.34,126.42,126.74,128.28,128.32,128.40,131.12,131.61,132.49$, 135.43, 145.30, 149.13, 161.28; IR (KBr) v 3327, 3063, 3028, 2963, 2926, 2833, 1661, 1607, 1576, 1537, 1493, 1450, 1368, 1352, 1279, 1244, 1209, 1107, 1059, 1032, 957, 852, 762, 736, 700, 681, $650 \mathrm{~cm}^{-1} ; \mathrm{HRMS} m / z[\mathrm{M}+\mathrm{H}]^{+}$calcd. for $\left[\mathrm{C}_{24} \mathrm{H}_{24} \mathrm{~N}_{3} \mathrm{O}_{3}\right]^{+} 402.1812$, found 402.1812 .

(S)-N-(((4S,5R)-2-(4-chlorophenyl)-5-phenyl-4,5-dihydrooxazol-4-yl)methyl)-1-phenylethana mine (4f)

Yellow oil, 79\% yield, $[\alpha]_{\mathrm{D}}{ }^{20}=-171.0\left(\mathrm{c} 1.0 \mathrm{CH}_{3} \mathrm{COOC}_{2} \mathrm{H}_{5}\right) ;{ }^{1} \mathrm{H}$ NMR $(400 \mathrm{MHz}$, $\left.\mathrm{CDCl}_{3}\right) \delta=7.94(\mathrm{~d}, J=8.5 \mathrm{~Hz}, 2 \mathrm{H}), 7.39(\mathrm{~d}, J=8.5 \mathrm{~Hz}, 2 \mathrm{H}), 7.36-7.08(\mathrm{~m}, 10 \mathrm{H}), 5.76(\mathrm{~d}$, $J=10.0 \mathrm{~Hz}, 1 \mathrm{H}), 4.64(\mathrm{dt}, J=9.9,7.0 \mathrm{~Hz}, 1 \mathrm{H}), 3.42(\mathrm{q}, J=6.5 \mathrm{~Hz}, 1 \mathrm{H}), 2.28(\mathrm{~d}, J=7.0 \mathrm{~Hz}$, $2 \mathrm{H}), 1.57$ (br s, $1 \mathrm{H}), 1.12(\mathrm{~d}, J=6.6 \mathrm{~Hz}, 3 \mathrm{H}) ;{ }^{13} \mathrm{C} \mathrm{NMR}\left(100 \mathrm{MHz}, \mathrm{CDCl}_{3}\right) \delta=23.95,49.39$, 58.22, 70.06, 83.44, 126.12, 126.37, 126.43, 126.78, 128.26, 128.35, 128.75, 129.77, 136.33, 137.77, 145.45, 162.82; IR (KBr) v 3318, 3061, 3028, 2965, 2924, 1651, 1599, 1491, 1452, 1402, 1368, 1333, 1277, 1244, 1170, 1128, 1092, 1074, 1015, 964, 841, 760, 731, 700, 677, 550, $534 \mathrm{~cm}^{-1}$; HRMS $m / z[\mathrm{M}+\mathrm{H}]^{+}$calcd. for $\left[\mathrm{C}_{24} \mathrm{H}_{24} \mathrm{ClN}_{2} \mathrm{O}\right]^{+} 391.1572$, found 391.1572.

(S)-N-(((4S,5R)-2-(2-chlorophenyl)-5-phenyl-4,5-dihydrooxazol-4-yl)methyl)-1-phenylethana mine $(4 \mathrm{~g})$

Yellow oil, 84.8\% yield, $[\alpha]_{\mathrm{D}}{ }^{20}=-259.2\left(\mathrm{c} 1.0 \mathrm{CH}_{3} \mathrm{COOC}_{2} \mathrm{H}_{5}\right) ;{ }^{1} \mathrm{H}$ NMR $(400 \mathrm{MHz}$, $\left.\mathrm{CDCl}_{3}\right) \delta=7.80(\mathrm{dd}, J=7.7,1.6 \mathrm{~Hz}, 1 \mathrm{H}), 7.46(\mathrm{dd}, J=8.0,0.8 \mathrm{~Hz}, 1 \mathrm{H}), 7.39-7.06(\mathrm{~m}, 12 \mathrm{H})$, $5.79(\mathrm{~d}, J=10.1 \mathrm{~Hz}, 1 \mathrm{H}), 4.69(\mathrm{dt}, J=10.0,6.9 \mathrm{~Hz}, 1 \mathrm{H}), 3.43(\mathrm{q}, J=6.6 \mathrm{~Hz}, 1 \mathrm{H}), 2.39-2.20(\mathrm{~m}$, 2H), 1.54 (br s, $1 \mathrm{H}), 1.13(\mathrm{~d}, J=6.6 \mathrm{~Hz}, 3 \mathrm{H}) ;{ }^{13} \mathrm{C} \mathrm{NMR}\left(100 \mathrm{MHz}, \mathrm{CDCl}_{3}\right) \delta=24.05,49.39$, $58.18,70.12,83.55,126.43,126.44,126.63,126.72,127.45,128.17,128.30,128.32,130.77,131.45$, 131.74, 133.53, 136.05, 145.37, 162.47; IR (KBr) v 3323, 3061, 3028, 2961, 1651, 1593, 1493, 
$1477,1452,1435,1368,1331,1279,1240,1132,1096,1036,961,914,762,735,700,654 \mathrm{~cm}^{-1}$; HRMS $m / z[\mathrm{M}+\mathrm{H}]^{+}$calcd. for $\left[\mathrm{C}_{24} \mathrm{H}_{24} \mathrm{ClN}_{2} \mathrm{O}\right]^{+} 391.1572$, found 391.1575 .

(S)-1-phenyl-N-(((4S,5R)-5-phenyl-2-(pyridin-4-yl)-4,5-dihydrooxazol-4-yl)methyl)ethanamine (4h)

Yellow oil, $82 \%$ yield, $[\alpha]_{\mathrm{D}}{ }^{20}=-302.7\left(\mathrm{c} 1.0 \mathrm{CH}_{3} \mathrm{COOC}_{2} \mathrm{H}_{5}\right) ;{ }^{1} \mathrm{H}$ NMR $(400 \mathrm{MHz}$, $\left.\mathrm{CDCl}_{3}\right) \delta=8.74(\mathrm{~d}, J=5.9 \mathrm{~Hz}, 2 \mathrm{H}), 7.85(\mathrm{~d}, J=5.9 \mathrm{~Hz}, 2 \mathrm{H}), 7.40-7.09(\mathrm{~m}, 10 \mathrm{H}), 5.82(\mathrm{~d}$, $J=10.1 \mathrm{~Hz}, 1 \mathrm{H}), 4.70(\mathrm{dt}, J=10.0,7.1 \mathrm{~Hz}, 1 \mathrm{H}), 3.44(\mathrm{q}, J=6.5 \mathrm{~Hz}, 1 \mathrm{H}), 2.39-2.20(\mathrm{~m}, 2 \mathrm{H})$, $1.97(\mathrm{br} \mathrm{s}, 1 \mathrm{H}), 1.15(\mathrm{~d}, J=6.6 \mathrm{~Hz}, 3 \mathrm{H}) ;{ }^{13} \mathrm{C}$ NMR $\left(100 \mathrm{MHz}, \mathrm{CDCl}_{3}\right) \delta=23.78,49.16,58.22$, $70.09,83.63,122.05,126.30,126.41,126.88,128.37,128.42,134.88,135.83,145.06,150.38$, 162.03; IR (KBr) v 3061, 3030, 2963, 2924, 1655, 1599, 1558, 1493, 1452, 1410, 1368, 1339, 1277, 1210, 1128, 1094, 1080, 1063, 991, 961, 837, 760, 745, 700, $681 \mathrm{~cm}^{-1}$; HRMS m/z [M + H] ${ }^{+}$ calcd. for $\left[\mathrm{C}_{23} \mathrm{H}_{24} \mathrm{~N}_{3} \mathrm{O}\right]^{+} 358.1914$, found 358.1914.

(S)-1-phenyl-N-(((4S,5R)-5-phenyl-2-(pyridin-2-yl)-4,5-dihydrooxazol-4-yl)methyl)ethanamine (4i)

Yellow oil, $87 \%$ yield, $[\alpha]_{\mathrm{D}}{ }^{20}=-261.0\left(\mathrm{c} 1.0 \mathrm{CH}_{3} \mathrm{COOC}_{2} \mathrm{H}_{5}\right) ;{ }^{1} \mathrm{H}$ NMR $(400 \mathrm{MHz}$, $\left.\mathrm{CDCl}_{3}\right) \delta=8.74(\mathrm{~d}, J=4.2 \mathrm{~Hz}, 1 \mathrm{H}), 8.06(\mathrm{~d}, J=7.9 \mathrm{~Hz}, 1 \mathrm{H}), 7.79(\mathrm{td}, J=7.8,1.6 \mathrm{~Hz}, 1 \mathrm{H})$, 7.48-7.35 (m, 1H), 7.35-7.01 (m, 10H), $5.85(\mathrm{~d}, J=10.1 \mathrm{~Hz}, 1 \mathrm{H}), 4.89-4.67(\mathrm{~m}, 1 \mathrm{H}), 3.52(\mathrm{q}, J$ $=6.5 \mathrm{~Hz}, 1 \mathrm{H}), 3.25(\mathrm{~s}, 1 \mathrm{H}), 2.43-2.12(\mathrm{~m}, 2 \mathrm{H}), 1.19(\mathrm{~d}, J=6.6 \mathrm{~Hz}, 3 \mathrm{H}) ;{ }^{13} \mathrm{C} \mathrm{NMR}(100 \mathrm{MHz}$, $\left.\mathrm{CDCl}_{3}\right) \delta=23.31,49.11,58.32,69.63,83.76,124.09,125.81,126.36,126.59,127.01,128.30$, $128.34,128.36,135.87,136.79,144.34,146.37,150.03,162.96$; IR $(\mathrm{KBr}) \vee 3314,3059,3028$, 2961, 2924, 1653, 1647, 1582, 1570, 1493, 1468, 1452, 1439, 1368, 1341, 1246, 1117, 1098, 1043, 993, 962, 799, 745, 700, $621 \mathrm{~cm}^{-1}$; HRMS $m / z[\mathrm{M}+\mathrm{H}]^{+}$calcd. for $\left[\mathrm{C}_{23} \mathrm{H}_{24} \mathrm{~N}_{3} \mathrm{O}\right]^{+} 358.1914$, found 358.1913 .

2-((4S,5R)-5-phenyl-4-(((S)-1-phenylethyl)amino)methyl)-4,5-dihydrooxazol-2-yl)phenol (4j)

White solid, 87\% yield, mp:76 79 ${ }^{\circ} \mathrm{C},[\alpha]_{\mathrm{D}}{ }^{20}=-256.6\left(\mathrm{c} 1.0 \mathrm{CH}_{3} \mathrm{COOC}_{2} \mathrm{H}_{5}\right) ;{ }^{1} \mathrm{H} \mathrm{NMR}$ $\left(400 \mathrm{MHz}, \mathrm{CDCl}_{3}\right) \delta=11.96(\mathrm{~s}, 1 \mathrm{H}), 7.74(\mathrm{dd}, J=7.8,1.5 \mathrm{~Hz}, 1 \mathrm{H}), 7.43-7.14(\mathrm{~m}, 9 \mathrm{H}), 7.11$ $(\mathrm{d}, J=7.0 \mathrm{~Hz}, 2 \mathrm{H}), 7.03(\mathrm{~d}, J=8.3 \mathrm{~Hz}, 1 \mathrm{H}), 6.87(\mathrm{t}, J=7.3 \mathrm{~Hz}, 1 \mathrm{H}), 5.75(\mathrm{~d}, J=9.9 \mathrm{~Hz}, 1 \mathrm{H})$, $4.68(\mathrm{dt}, J=9.8,6.9 \mathrm{~Hz}, 1 \mathrm{H}), 3.39(\mathrm{q}, J=6.6 \mathrm{~Hz}, 1 \mathrm{H}), 2.34-2.22(\mathrm{~m}, 2 \mathrm{H}), 1.11(\mathrm{~d}, J=6.6 \mathrm{~Hz}$, $3 \mathrm{H}) ;{ }^{13} \mathrm{C}$ NMR $\left(100 \mathrm{MHz}, \mathrm{CDCl}_{3}\right) \delta=23.89,49.25,58.14,68.82,82.51,110.43,116.85,118.84$, $126.36,126.49,126.93,128.31,128.46,128.53,133.70,135.59,145.18,160.00,165.44 ;$ IR (KBr) v 3061, 3028, 2961, 2924, 2849, 1643, 1616, 1491, 1454, 1368, 1352, 1312, 1258, 1231, 1155, 1130, 1070, 1032, 961, 756, 700, 571, $540 \mathrm{~cm}^{-1}$; HRMS m/z [M + H] ${ }^{+}$calcd. for $\left[\mathrm{C}_{24} \mathrm{H}_{25} \mathrm{~N}_{2} \mathrm{O}_{2}\right]^{+}$ 373.1911, found 373.1909.

2-((4S,5R)-5-phenyl-4-(((S)-1-phenylethyl)amino)methyl)-4,5-dihydrooxazol-2-yl)aniline (4k)

Yellow oil, $82 \%$ yield, $[\alpha]_{\mathrm{D}}{ }^{20}=-285.7\left(\mathrm{c} 1.0 \mathrm{CH}_{3} \mathrm{COOC}_{2} \mathrm{H}_{5}\right) ;{ }^{1} \mathrm{H}$ NMR $(400 \mathrm{MHz}$, $\left.\mathrm{CDCl}_{3}\right) \delta=7.79(\mathrm{~d}, J=7.1 \mathrm{~Hz}, 1 \mathrm{H}), 7.35-7.11(\mathrm{~m}, 11 \mathrm{H}), 6.66(\mathrm{dd}, J=13.6,7.6 \mathrm{~Hz}, 2 \mathrm{H}), 6.04(\mathrm{~s}$, $2 \mathrm{H}), 5.65(\mathrm{~d}, J=9.9 \mathrm{~Hz}, 1 \mathrm{H}), 4.68(\mathrm{dt}, J=9.7,7.1 \mathrm{~Hz}, 1 \mathrm{H}), 3.41(\mathrm{q}, J=6.5 \mathrm{~Hz}, 1 \mathrm{H}), 2.36-2.13$ $(\mathrm{m}, 2 \mathrm{H}), 1.44(\mathrm{br} \mathrm{s}, 1 \mathrm{H}), 1.11(\mathrm{~d}, J=6.6 \mathrm{~Hz}, 3 \mathrm{H}) ;{ }^{13} \mathrm{C} \mathrm{NMR}\left(100 \mathrm{MHz}, \mathrm{CDCl}_{3}\right) \delta=23.91$, $49.76,58.21,70.06,81.32,108.70,115.75,116.14,126.46,126.49,126.85,128.35,128.41,129.91$, 132.37, 136.66, 145.54, 148.80, 164.07; IR (KBr) v 3466, 3296, 3061, 3028, 2961, 2924, 1636, 1609, 1595, 1560, 1490, 1454, 1368, 1348, 1319, 1263, 1161, 1078, 1053, 970, 914, 750, 700, 569, $538 \mathrm{~cm}^{-1}$; HRMS m/z [M + H] $]^{+}$calcd. for $\left[\mathrm{C}_{24} \mathrm{H}_{26} \mathrm{~N}_{3} \mathrm{O}\right]^{+}$372.2070, found 372.2070.

2-((4S,5R)-5-phenyl-4-((((S)-1-phenylethyl)amino)methyl)-4,5-dihydrooxazol-2-yl)naphthalen$1-\mathrm{ol}(41)$

Dark green solid, $88 \%$ yield, mp: 79 81 ${ }^{\circ} \mathrm{C},[\alpha]_{\mathrm{D}}{ }^{20}=+166.9\left(\mathrm{c} 1.0 \mathrm{CH}_{3} \mathrm{COOC}_{2} \mathrm{H}_{5}\right) ;{ }^{1} \mathrm{H}$ NMR $\left(400 \mathrm{MHz}, \mathrm{CDCl}_{3}\right) \delta=13.11(\mathrm{br} \mathrm{s}, 1 \mathrm{H}), 8.42(\mathrm{~d}, J=8.2 \mathrm{~Hz}, 1 \mathrm{H}), 7.77(\mathrm{~d}, J=7.8 \mathrm{~Hz}$, $1 \mathrm{H}), 7.72(\mathrm{~d}, J=8.7 \mathrm{~Hz}, 1 \mathrm{H}), 7.57(\mathrm{td}, J=7.0,1.4 \mathrm{~Hz}, 1 \mathrm{H}), 7.51(\mathrm{td}, J=6.9,1.3 \mathrm{~Hz}, 1 \mathrm{H})$, $7.40-7.05(\mathrm{~m}, 11 \mathrm{H}), 5.81(\mathrm{~d}, J=9.8 \mathrm{~Hz}, 1 \mathrm{H}), 4.74(\mathrm{dt}, J=9.8,6.9 \mathrm{~Hz}, 1 \mathrm{H}), 3.43(\mathrm{q}, J=6.6 \mathrm{~Hz}$, 
1H), 2.37-2.28 (m, 2H), $1.13(\mathrm{~d}, J=6.6 \mathrm{~Hz}, 3 \mathrm{H}) ;{ }^{13} \mathrm{C} \mathrm{NMR}\left(100 \mathrm{MHz}, \mathrm{CDCl}_{3}\right) \delta=23.87$, $49.32,58.13,68.52,82.68,103.37,118.20,123.47,123.70,124.75,125.54,126.37,126.51,126.90$, $127.54,128.44,128.50,128.52,135.62,136.28,145.18,158.85,166.38$; IR (KBr) v 3061, 3030, 2959, 2924, 2851, 1628, 1599, 1578, 1466, 1450, 1408, 1391, 1304, 1260, 1140, 1078, 964, 810, $756,700 \mathrm{~cm}^{-1}$; HRMS $m / z[\mathrm{M}+\mathrm{H}]^{+}$calcd. for $\left[\mathrm{C}_{28} \mathrm{H}_{27} \mathrm{~N}_{2} \mathrm{O}_{2}\right]^{+} 423.2067$, found 423.2064 .

(S)-1-phenyl-N-(((4S,5R)-5-phenyl-2-(thiophen-2-yl)-4,5-dihydrooxazol-4-yl)methyl)ethana mine $(4 \mathrm{~m})$

Yellow oil, 90\% yield, $[\alpha]_{\mathrm{D}}{ }^{20}=-256.6\left(\mathrm{c} 1.0 \mathrm{CH}_{3} \mathrm{COOC}_{2} \mathrm{H}_{5}\right) ;{ }^{1} \mathrm{H} \mathrm{NMR}(400 \mathrm{MHz}$, $\left.\mathrm{CDCl}_{3}\right) \delta=7.67(\mathrm{~d}, J=2.2 \mathrm{~Hz}, 1 \mathrm{H}), 7.48(\mathrm{~d}, J=4.5 \mathrm{~Hz}, 1 \mathrm{H}), 7.41-7.03(\mathrm{~m}, 11 \mathrm{H}), 5.77(\mathrm{~d}$, $J=9.8 \mathrm{~Hz}, 1 \mathrm{H}), 4.62(\mathrm{q}, J=7.1 \mathrm{~Hz}, 1 \mathrm{H}), 3.51-3.27(\mathrm{~m}, 1 \mathrm{H}), 2.34-2.24(\mathrm{~m}, 2 \mathrm{H}), 1.53(\mathrm{br} \mathrm{s}, 1 \mathrm{H})$, $1.11(\mathrm{~d}, J=6.4 \mathrm{~Hz}, 3 \mathrm{H}) ;{ }^{13} \mathrm{C}$ NMR $\left(100 \mathrm{MHz}, \mathrm{CDCl}_{3}\right) \delta=24.01,49.28,58.21,70.05,83.67$, $126.43,126.74,127.71,128.26,128.33,130.19,130.21,130.66,136.18,145.38,159.54 ; \mathrm{IR}(\mathrm{KBr})$ v $3323,3082,3061,3026,2963,2924,1649,1522,1493,1452,1433,1369,1329,1277,1217$, $1128,1082,1059,1018,959,851,760,716,700,650 \mathrm{~cm}^{-1}$; HRMS m/z [M + H] $]^{+}$calcd. for $\left[\mathrm{C}_{22} \mathrm{H}_{23} \mathrm{~N}_{2} \mathrm{OS}\right]^{+}$363.1526, found 363.1526.

(S)-N-(((4S,5R)-2-(furan-2-yl)-5-phenyl-4,5-dihydrooxazol-4-yl)methyl)-1-phenylethanamine (4n)

White solid, $84 \%$ yield, $\mathrm{mp}: 45 \sim 48{ }^{\circ} \mathrm{C} ;[\alpha]_{\mathrm{D}}^{20}=-279.1\left(\mathrm{c} 1.0 \mathrm{CH}_{3} \mathrm{COOC}_{2} \mathrm{H}_{5}\right) ;{ }^{1} \mathrm{H}$ NMR $\left(400 \mathrm{MHz}, \mathrm{CDCl}_{3}\right) \delta=7.57(\mathrm{~s}, 1 \mathrm{H}), 7.42-7.09(\mathrm{~m}, 10 \mathrm{H}), 7.02(\mathrm{~d}, J=3.1 \mathrm{~Hz}, 1 \mathrm{H}), 6.51(\mathrm{~s}, 1 \mathrm{H})$, $5.74(\mathrm{~d}, J=9.9 \mathrm{~Hz}, 1 \mathrm{H}), 4.69-4.69(\mathrm{~m}, 1 \mathrm{H}), 3.45(\mathrm{q}, J=6.4 \mathrm{~Hz}, 1 \mathrm{H}), 2.29(\mathrm{~d}, J=6.9 \mathrm{~Hz}, 2 \mathrm{H})$, 2.00 (br s, $1 \mathrm{H}), 1.13(\mathrm{~d}, J=6.5 \mathrm{~Hz}, 3 \mathrm{H}) ;{ }^{13} \mathrm{C}$ NMR $\left(100 \mathrm{MHz}, \mathrm{CDCl}_{3}\right) \delta=23.70,49.24,58.20$, $69.69,83.35,111.63,114.90,126.38,126.47,126.80,128.30,128.34,135.96,142.71,145.12$, 145.56, 156.15; IR (KBr) v 3318, 3061, 3028, 2963, 2924, 2855, 1674, 1580, 1560, 1493, 1481, 1452, 1402, 1331, 1229, 1169, 1092, 1011, 962, 885, 754, 700, 631, 596, $552 \mathrm{~cm}^{-1}$; HRMS m/z $[\mathrm{M}+\mathrm{H}]^{+}$calcd. for $\left[\mathrm{C}_{22} \mathrm{H}_{23} \mathrm{~N}_{2} \mathrm{O}_{2}\right]^{+}$347.1754, found 347.1754.

(S)-N-((4S,5R)-2-methyl-5-phenyl-4,5-dihydrooxazol-4-yl)methyl)-1-phenylethanamine (4o)

Yellow solid, $85 \%$ yield, mp: $44 \sim 47^{\circ} \mathrm{C} ;[\alpha]_{\mathrm{D}}^{20}=-252.6\left(\right.$ c $\left.1.0 \mathrm{CH}_{3} \mathrm{COOC}_{2} \mathrm{H}_{5}\right) ;{ }^{1} \mathrm{H}$ $\operatorname{NMR}\left(400 \mathrm{MHz}, \mathrm{CDCl}_{3}\right) \delta=7.37-7.04(\mathrm{~m}, 10 \mathrm{H}), 5.57(\mathrm{~d}, J=10.0 \mathrm{~Hz}, 1 \mathrm{H}), 4.44-4.37(\mathrm{~m}, 1 \mathrm{H})$, $3.39(\mathrm{q}, J=6.5 \mathrm{~Hz}, 1 \mathrm{H}), 2.24-2.10(\mathrm{~m}, 2 \mathrm{H}), 2.07(\mathrm{~d}, J=0.8 \mathrm{~Hz}, 3 \mathrm{H}), 1.10(\mathrm{~d}, J=6.6 \mathrm{~Hz}, 3 \mathrm{H})$; ${ }^{13} \mathrm{C}$ NMR $\left(100 \mathrm{MHz}, \mathrm{CDCl}_{3}\right) \delta=14.06,23.82,49.41,58.14,69.48,83.12,126.24,126.40,126.69$, $128.05,128.25,136.43,145.43,164.76$; IR (KBr) v 3320, 3061, 3026, 2967, 2928, 1680, 1603, $1493,1452,1387,1369,1310,1229,1130,1078,1028,974,914,760,700,623,592,538 \mathrm{~cm}^{-1}$; HRMS $m / z[\mathrm{M}+\mathrm{H}]^{+}$calcd. for $\left[\mathrm{C}_{19} \mathrm{H}_{23} \mathrm{~N}_{2} \mathrm{O}\right]^{+}$295.1805, found 295.1801.

(S)-N-(((4S,5R)-2-benzyl-5-phenyl-4,5-dihydrooxazol-4-yl)methyl)-1-phenylethana mine (4p)

White solid, 83\% yield, mp: $103 \sim 106{ }^{\circ} \mathrm{C},[\alpha]_{\mathrm{D}}{ }^{20}=-248.0\left(\mathrm{c} 1.0 \mathrm{CH}_{3} \mathrm{COOC}_{2} \mathrm{H}_{5}\right) ;{ }^{1} \mathrm{H}$ NMR $\left(400 \mathrm{MHz}, \mathrm{CDCl}_{3}\right) \delta=7.44-7.15(\mathrm{~m}, 11 \mathrm{H}), 7.08-7.02(\mathrm{~m}, 4 \mathrm{H}), 5.56(\mathrm{~d}, J=10.0 \mathrm{~Hz}, 1 \mathrm{H})$, $4.47-4.40(\mathrm{~m}, 1 \mathrm{H}), 3.69(\mathrm{q}, J=14.6 \mathrm{~Hz}, 2 \mathrm{H}), 3.37(\mathrm{q}, J=6.5 \mathrm{~Hz}, 1 \mathrm{H}), 2.21-2.05(\mathrm{~m}, 2 \mathrm{H}), 1.52$ (br s, $1 \mathrm{H}), 1.10(\mathrm{~d}, J=6.6 \mathrm{~Hz}, 3 \mathrm{H}) ;{ }^{13} \mathrm{C}$ NMR $\left(100 \mathrm{MHz}, \mathrm{CDCl}_{3}\right) \delta=23.99,35.08,49.41,58.17$, 69.41, 83.25, 126.20, 126.40, 126.72, 127.10, 128.00, 128.19, 128.30, 128.65, 129.06, 135.07, 136.32, 145.33, 166.20; IR (KBr) v 3302, 3057, 3028, 2985, 2932, 2857, 1663, 1603, 1558, 1495, $1449,1267,1244,1219,1167,1142,1126,1076,1055,1028,993,972,920,822,784,736,723$, $700,575,538 \mathrm{~cm}^{-1}$; HRMS $\mathrm{m} / z[\mathrm{M}+\mathrm{H}]^{+}$calcd. for $\left[\mathrm{C}_{25} \mathrm{H}_{27} \mathrm{~N}_{2} \mathrm{O}\right]^{+} 371.2118$, found 371.2118.

\section{Conclusions}

In conclusion, we have developed a new isomerization of amides 3 that leads to 2-oxzaolines 4 in a completely highly regio- and stereoselective isomerization manner. Further application of these 2-oxzaolines in catalytic asymmetric reaction is currently underway in our laboratory. 
Supplementary Materials: The supplementary materials in the text are available online. CCDC 2059257 contain the supplementary crystallographic data for this paper. These data can be obtained free of charge via www.ccdc.cam.ac.uk/data_request/cif (accessed on 6 February 2021), or by emailing data_request@ccdc.cam.ac.uk, or by contacting The Cambridge Crystallographic Data Centre, 12 Union Road, Cambridge CB2 1EZ, UK; fax: +44-1223-336033.

Author Contributions: Z.Z. and X.Z. designed the chemical synthesis, analyzed the results, and wrote the manuscript. X.Z. and B.M. performed the chemical synthesis experiments and analyzed the results. All authors have read and agreed to the published version of the manuscript.

Funding: This research received no external funding.

Institutional Review Board Statement: Not applicable.

Informed Consent Statement: Not applicable.

Data Availability Statement: The data presented in this study are available in this article.

Conflicts of Interest: The authors declare no conflict of interest.

Sample Availability: Not available.

\section{References}

1. Wong, G.S.K.; Wu, W. 2-oxazolines. Chem. Heterocycl. Compd. 2004, 60, 331-528.

2. Wipf, P.; Miller, C.P. Total synthesis of westiellamide. J. Am. Chem. Soc. 1992, 114, 10975-10977. [CrossRef]

3. Xiao, S.-J.; Guo, D.-L.; Zhang, M.-S.; Chen, F.; Ding, L.-S.; Zhou, Y. Three new oxazoline alkaloids from Gymnotheca chinensis. J. Asian Nat. Prod. Res. 2016, 18,719-723. [CrossRef] [PubMed]

4. Campiani, G.; De Angelis, M.; Armaroli, S.; Fattorusso, C.; Catalanotti, B.; Ramunno, A.; Nacci, V.; Novellino, E.; Grewer, C.; Ionescu, D.; et al. A rational approach to the design of selective substrates and potent nontransportable inhibitors of the excitatory amino acid transporter EAAC1 (EAAT3). New glutamate and aspartate analogues as potential neuroprotective agents. J. Med. Chem. 2001, 44, 2507-2510. [CrossRef] [PubMed]

5. Avalos-Alanis, F.G.; Hernandez-Fernandez, E.; Carranza-Rosales, P.; Lopez-Cortina, S.; Hernandez-Fernandez, J.; Ordonez, M.; Guzman-Delgado, N.E.; Morales-Vargas, A.; Velazquez-Moreno, V.M.; Santiago-Mauricio, M.G. Synthesis, antimycobacterial and cytotoxic activity of $\alpha, \beta$-unsaturated amides and 2,4-disubstituted oxazoline derivatives. Bioorg. Med. Chem. Lett. 2017, 27, 821-825. [CrossRef] [PubMed]

6. Li, Q.; Woods, K.W.; Claiborne, A.; Gwaltney, S.L., II; Barr, K.J.; Liu, G.; Gehrke, L.; Credo, R.B.; Hui, Y.H.; Lee, J.; et al. Synthesis and Biological Evaluation of 2-Indolyloxazolines as a New Class of Tubulin Polymerization Inhibitors. Discovery of A-289099 as an Orally Active Antitumor Agent. Bioorg. Med. Chem. Lett. 2002, 12, 465-469. [CrossRef]

7. Ye, X.-Y.; Liang, Z.-Q.; Jin, C.; Lang, Q.-W.; Chen, G.-Q.; Zhang, X. Design of oxa-spirocyclic PHOX ligands for the asymmetric synthesis of lorcaserin via iridium-catalyzed asymmetric hydrogenation. Chem. Commun. 2021, 57, 195-198. [CrossRef]

8. Xi, C.-C.; Zhao, X.-J.; Tian, J.-M.; Chen, Z.-M.; Zhang, K.; Zhang, F.-M.; Tu, Y.-Q.; Dong, J.-W. Atroposelective Synthesis of Axially Chiral 3-Arylindoles by Copper-Catalyzed Asymmetric Cross-Coupling of Indoles with Quinones and Naphthoquinones. Org. Lett. 2020, 22, 4995-5000. [CrossRef]

9. Park, J.-U.; Ahn, H.-I.; Cho, H.-J.; Xuan, Z.; Kim, J.H. Asymmetric Synthesis of N-Fused 1,3-Oxazolidines via Pd-Catalyzed Decarboxylative (3+2) Cycloaddition. Adv. Synth. Catal. 2020, 362, 1836-1840. [CrossRef]

10. Qiu, Z.; Sun, R.; Yang, K.; Teng, D. Spiro indan-based phosphine-oxazolines as highly efficient P,N ligands for enantioselective Pd-catalyzed allylic alkylation of indoles and allylic etherification. Molecules 2019, 24, 1575. [CrossRef] [PubMed]

11. Itoh, K.; Sibi, M.P. Dibenzofuran-4,6-bis(oxazoline) (DBFOX). A novel trans-chelating bis(oxazoline) ligand for asymmetric reactions. Org. Biomol. Chem. 2018, 16, 5551-5565. [CrossRef]

12. Frump, J.A. Oxazolines. Their preparation, reactions, and applications. Chem. Rev. 1971, 71, 483-506. [CrossRef]

13. Goud, D.R.; Pathak, U. A mild and efficient synthesis of 2-oxazolines via transamidation-cyclodehydrosulfurization of thioamides with 2-aminoethanol. Synthesis 2012, 44, 3678-3682. [CrossRef]

14. Brandstatter, M.; Roth, F.; Luedtke, N.W. Synthesis of 2-Oxazolines by in Situ Desilylation and Cyclodehydration of $\beta$ Hydroxyamides. J. Org. Chem. 2015, 80, 40-51. [CrossRef]

15. Huang, H.; Yang, W.; Chen, Z.; Lai, Z.; Sun, J. A mild catalytic synthesis of 2-oxazolines via oxetane ring-opening: Rapid access to a diverse family of natural products. Chem. Sci. 2019, 10, 9586-9590. [CrossRef] [PubMed]

16. Petersson, M.J.; Jenkins, I.D.; Loughlin, W.A. The use of phosphonium anhydrides for the synthesis of 2-oxazolines, 2-thiazolines and 2-dihydrooxazine under mild conditions. Org. Biomol. Chem. 2009, 7, 739-746. [CrossRef]

17. Sharma, R.; Vadivel, S.K.; Duclos, R.I.; Makriyannis, A. Open vessel mode microwave-assisted synthesis of 2-oxazolines from carboxylic acids. Tetrahedron Lett. 2009, 50, 5780-5782. [CrossRef] [PubMed]

18. Rajaram, S.; Sigman, M.S. Modular Synthesis of Amine-Functionalized Oxazolines. Org. Lett. 2002, 4, 3399-3401. [CrossRef] [PubMed] 
19. Zhou, X.; Xu, X.; Li, Y.; Zhang, Z.; Zheng, Z.-b. Synthesis of chiral 4,5-dihydrothiazol-2-amines and 4,5-dihydrooxazol-2-amines through an unexpected ring opening reaction of azetidines. Tetrahedron Lett. 2016, 57, 1236-1238. [CrossRef]

20. Zhang, Z.; Bai, X.; Liu, R.; Zi, G. Synthesis of new chiral cis-3-aminoazetidines and their use in catalytic asymmetric reactions. Inorg. Chim. Acta 2009, 362, 1687-1691. [CrossRef]

21. Higgins, R.H.; Faircloth, W.J.; Baughman, R.G.; Eaton, Q.L. Ring Opening of Azetidinols by Phenols: Regiochemistry and Stereochemistry. J. Org. Chem. 1994, 59, 2172-2178. [CrossRef]

22. Purkarthofer, T.; Gruber, K.; Gruber-Khadjawi, M.; Waich, K.; Skranc, W.; Mink, D.; Griengl, H. A biocatalytic Henry reaction-The hydroxynitrile lyase from Hevea brasiliensis also catalyzes nitroaldol reactions. Angew. Chem. Int. Ed. 2006, 45, 3454-3456. [CrossRef] [PubMed]

23. Nishimura, M.; Minakata, S.; Takahashi, T.; Oderaotoshi, Y.; Komatsu, M. Asymmetric N1 Unit Transfer to Olefins with a Chiral Nitridomanganese Complex: Novel Stereoselective Pathways to Aziridines or Oxazolines. J. Org. Chem. 2002, 67, 2101-2110. [CrossRef] [PubMed] 